\title{
FRET in Dyads with Orthogonal Chromophores and Minimal Spectral Overlap*
}

\author{
Heinz Langhals ${ }^{* a}$ and Andreas Walter ${ }^{a}$ \\ aLMU University of Munich, Department of Chemistry, \\ Butenandtstraße 13, D-81377 Munich, Germany
}

Supporting Information

List of Contents

1. Experimental Page S2

1.1 Materials $\quad$ Page S2

1.2 Methods Page S11

2. References Page S11 


\section{Experimental}

\subsection{Materials}

Reference Materials: Fluorescence quantum yields $\Phi$ were determined by means of reference materials: (i) S-131 (CAS registry number RN 110590-84-6), $\Phi=1.0$; (ii) 3,4,9,10-perylenetetramethylester ${ }^{1}$ (RN 53159-49-2), $\Phi=1.0$; (iii) C25² (RN 335458-214), $\Phi=1.0$; S-19terrylenetetracarboxdiimide ${ }^{3}$ (RN 1029894-60-7), $\Phi=0.94$; peryleneterrylene dyad ${ }^{3}$ (RN 1313052-90-2), $\Phi=0.86$.

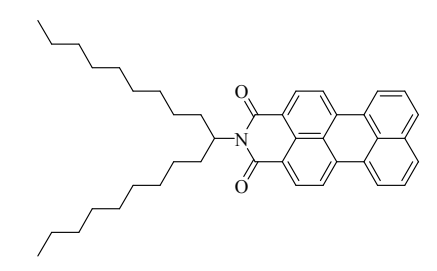

4

\section{2-(1-Nonyldecyl)-1H-benzo[5,10]anthra[2,1,9-def] isoquinoline-1,3(2H)-dione (7): ${ }^{4}$}

Copper powder $(7.43 \mathrm{~g}, 117 \mathrm{mmol})$ under argon atmosphere and 3-picoline $(500 \mathrm{~mL})$ were stirred at $90^{\circ} \mathrm{C}$ for $4 \mathrm{~h}$, treated with 9-(1-nonyldecyl)- $1 \mathrm{H}$ isochromeno[6',5',4':10,5,6]anthra[2,1,9-def]isoquinoline-1,3,8,10(9H)-tetraon ${ }^{5}$ (RN 130296-48-9) (15.3 g, $23.3 \mathrm{mmol}$ ) stirred at $175^{\circ} \mathrm{C}$ for and $10 \mathrm{~h}$, allowed to cool, precipitated with $2 \mathrm{M}$ aqueous $\mathrm{HCl}(1300 \mathrm{~mL})$, stirred at room temperature for $60 \mathrm{~min}$, collected by vacuum filtration (D4 glass filter), washed with $2 \mathrm{M}$ aqueous $\mathrm{HCl}(800 \mathrm{~mL}$ ) and distilled water $(2000 \mathrm{~mL})$, dried at $90^{\circ} \mathrm{C}$ for $2 \mathrm{~d}$ and purified by column separation (silica gel, chloroform). Yield $10.5 \mathrm{~g}\left(76 \%\right.$ ) bright red solid, m.p. $144^{\circ} \mathrm{C}$ (ref. ${ }^{4} 143-$ $143.5^{\circ} \mathrm{C}$ ). $R_{\mathrm{f}}$ value (silica gel, $\left.\mathrm{CHCl}_{3}\right): 0.8 . \mathrm{IR}(\mathrm{ATR}): \widetilde{v}=2952(\mathrm{~m}), 2923(\mathrm{~m}), 2855(\mathrm{~m})$, $2363(w), 1697(s), 1659(s), 1593(s), 1578(m), 1506(w), 1483(w), 1465(w), 1430$ $(w), 1404(w), 1352(m), 1340(s), 1305(w), 1252(m), 1202(w), 1175(w), 1125(w)$, $1106(w), 1018(w), 964(w), 854(w), 811(m), 747(m), 731(w), 694(w), 667 \mathrm{~cm}^{-1}(w)$. ${ }^{1} \mathrm{H} \mathrm{NMR}\left(600 \mathrm{MHz}, \mathrm{CDCl}_{3}, 27.0^{\circ} \mathrm{C}, \mathrm{TMS}\right): \delta=8.27\left(\mathrm{~d},{ }^{3} \mathrm{~J}(\mathrm{H}, \mathrm{H})=7.9 \mathrm{~Hz}, 2 \mathrm{H}, \mathrm{CH}_{\text {arom. }}\right)$, $7.97\left(\mathrm{~d},{ }^{3} \mathrm{~J}(\mathrm{H}, \mathrm{H})=7.6 \mathrm{~Hz}, 2 \mathrm{H}, \mathrm{CH}_{\text {arom }}\right), 7.92\left(\mathrm{~d},{ }^{3} \mathrm{~J}(\mathrm{H}, \mathrm{H})=7.6 \mathrm{~Hz}, 2 \mathrm{H}, \mathrm{CH}_{\text {arom }}\right), 7.63(\mathrm{~d}$, $\left.{ }^{3} \mathrm{~J}(\mathrm{H}, \mathrm{H})=8.0 \mathrm{~Hz}, 2 \mathrm{H}, \mathrm{CH}_{\text {arom. }}\right), 7.36\left(\mathrm{dd},{ }^{3} \mathrm{~J}(\mathrm{H}, \mathrm{H})=7.7 \mathrm{~Hz},{ }^{3} \mathrm{~J}(\mathrm{H}, \mathrm{H})=7.7 \mathrm{~Hz}, 2 \mathrm{H}\right.$, $\mathrm{CH}_{\text {arom. }}$ ), 5.21-5.16 (m, $\left.1 \mathrm{H}, \mathrm{CH}\right), 2.30-2.23\left(\mathrm{~m}, 2 \mathrm{H}, \beta-\mathrm{CH}_{2}\right), 1.94-1.88\left(\mathrm{~m}, 2 \mathrm{H}, \beta-\mathrm{CH}_{2}\right)$, 1.41-1.16 (m, $\left.28 \mathrm{H}, 14 \times \mathrm{CH}_{2}\right), 0.83 \mathrm{ppm}\left(\mathrm{t}, 3 \mathrm{~J}(\mathrm{H}, \mathrm{H})=7.1 \mathrm{~Hz}, 6 \mathrm{H}, 2 \times \mathrm{CH}_{3}\right) \cdot{ }^{13} \mathrm{C} \mathrm{NMR}$ $\left(150 \mathrm{MHz}, \mathrm{CDCl}_{3}, 27.0^{\circ} \mathrm{C}\right): \delta=165.0,164.0,136.3,134.2,131.7,130.6,130.4,129.4$, 128.7, 127.4, 126.6, 126.0, 123.0, 121.3, 120.5, 119.6, 54.3, 32.4, 31.9, 29.7, 29.6, 29.3, 27.1, 22.7, $14.1 \mathrm{ppm}$. UV/VIS $\left(\mathrm{CHCl}_{3}\right): \lambda_{\max }(\varepsilon)=482.8$ (31900), $506.2 \mathrm{~nm}(31500)$. Fluorescence $\left(\mathrm{CHCl}_{3}\right): \lambda_{\max }\left(I_{\text {rel }}\right)=537.9(1.00), 578.4 \mathrm{~nm}$ (0.83). Fluorescence quantum yield $\left(\mathrm{CHCl}_{3}, \lambda_{\mathrm{exc}}=482 \mathrm{~nm}, E_{482 \mathrm{~nm}, 1 \mathrm{~cm}}=0.0161\right.$, reference: $\mathrm{S}-13$ with $\left.\Phi=1.00\right): 0.95$. 
MS (DEP/EI) m/z (\%): $587(84)\left[M^{+}\right], 322(100)\left[M^{+}-\mathrm{C}_{19} \mathrm{H}_{38}\right]$. HRMS $\left(\mathrm{C}_{41} \mathrm{H}_{49} \mathrm{NO}_{2}\right) \mathrm{m} / \mathrm{z}$ : Calcd. 587.3763, found 587.3759; $\Delta=-0.0004 . \mathrm{C}_{41} \mathrm{H}_{49} \mathrm{NO}_{2}$ (587.4): Calcd. C 83.77, $\mathrm{H}$ 8.40, N 2.38; found C 83.78, H 8.48, N 2.33.

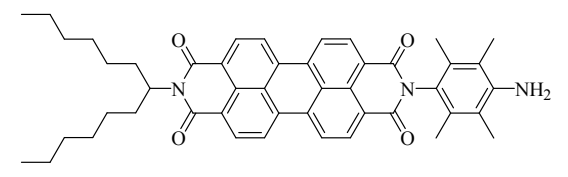

2-(4-Amino-2,3,5,6-tetramethylphenyl)-9-(1-hexylheptyl)anthra[2,1,9-def;6,5,10d'e'f']diisoquinoline-1,3,8,10(2H,9H)-tetraone:6 2,3,5,6-Tetramethylbenzene-1,4diamine $^{7}$ (RN 3102-87-2) (197 mg, 1.20 mmol), 9-(1-hexylheptyl)-1Hisochromeno[6',5',4':10,5,6]anthra[2,1,9-def]isoquinoline-1,3,8,10(9H)-tetraone (RN 130296-37-6) (230 mg, $400 \mu \mathrm{mol})$, zincacetate (14.8 mg, $80 \mu \mathrm{mol}$ ) and quinoline (4 mL) under argon atmosphere were stirred at $210^{\circ} \mathrm{C} 4 \mathrm{~h}$ (dark red mixture), allowed to cool, still warm and with vigorous stirring was dropped into $2 \mathrm{~m}$ aqueous $\mathrm{HCl}(300 \mathrm{~mL})$ stirred for $2 \mathrm{~h}$, allowed to stand for $16 \mathrm{~h}$, collected by vacuum filtration (D4 glass filter), washed with $2 \mathrm{~m}$ aqueous $\mathrm{HCl}(300 \mathrm{~mL})$, hot distilled water $(300 \mathrm{~mL})$ and a mixture of methanol and water $(300 \mathrm{~mL}, 1: 1)$, dried in vacuo at $110^{\circ} \mathrm{C}$ for $2 \mathrm{~d}$, purified by column separation (basic alumina, $\mathrm{CHCl}_{3} /$ methanol 80:1 and then silica gel, $\mathrm{CHCl}_{3} /$ methanol 40:1). Yield $250 \mathrm{mg}(87 \%)$ red solid, m.p. $>300^{\circ} \mathrm{C}$. $R_{\mathrm{f}}$ value (silica gel, $\mathrm{CHCl}_{3} /$ methanol 40:1): 0.4 . IR (ATR): $\widetilde{v}=3482(\mathrm{w}), 3395(\mathrm{w}), 2953(\mathrm{~m}), 2924(\mathrm{~m}), 2856(\mathrm{~m}), 2363(\mathrm{w}), 1698(\mathrm{~s})$, 1653 (s), 1592 (s), 1577 (s), 1507 (w), 1458 (w), 1432 (m), 1405 (m), 1346 (m), 1328 (s), $1250(\mathrm{~m}), 1198(\mathrm{w}), 1174(\mathrm{~m}), 1138(\mathrm{w}), 1107$ (w), 962 (w), 855 (w), $839(\mathrm{w}), 810$ (m), $802(\mathrm{w}), 748(\mathrm{~m}), 722(\mathrm{w}), 671 \mathrm{~cm}^{-1}(\mathrm{w}) .{ }^{1} \mathrm{H}$ NMR (600 MHz, $\left.\mathrm{CDCl}_{3}, 27.0^{\circ} \mathrm{C}, \mathrm{TMS}\right)$ : $\delta=8.78-8.66\left(\mathrm{~m}, 8 \mathrm{H}, \mathrm{CH}_{\text {arom. perylene }}\right), 5.22-5.16(\mathrm{~m}, 1 \mathrm{H}, \mathrm{CH}), 3.71\left(\mathrm{br}, 2 \mathrm{H}, \mathrm{NH}_{2}\right), 2.29$ $2.21\left(\mathrm{~m}, 2 \mathrm{H}, \beta-\mathrm{CH}_{2}\right), 2.16\left(\mathrm{~s}, 6 \mathrm{H}, 2 \times \mathrm{CH}_{3}\right), 2.06\left(\mathrm{~s}, 6 \mathrm{H}, 2 \times \mathrm{CH}_{3}\right), 1.91-1.83(\mathrm{~m}, 2 \mathrm{H}, \beta-$ $\left.\mathrm{CH}_{2}\right), 1.38-1.19(\mathrm{~m}, 16 \mathrm{H}), 0.83 \mathrm{ppm}\left(\mathrm{t},{ }^{3} \mathrm{~J}(\mathrm{H}, \mathrm{H})=6.7 \mathrm{~Hz}, 6 \mathrm{H}, 2 \times \mathrm{CH}_{3}\right) .{ }^{13} \mathrm{C} \mathrm{NMR}$ $\left(150 \mathrm{MHz}, \mathrm{CDCl}_{3}, 27.0^{\circ} \mathrm{C}\right): \delta=163.5,143.4,135.0,134.5,132.0,131.2,130.8,130.1$, 129.6, 126.8, 126.5, 124.3, 123.4, 123.2, 123.1, 118.9, 54.8, 32.4, 31.8, 29.2, 26.9, 22.6, 15.1, 14.0, 13.9 ppm. UV/VIS $\left(\mathrm{CHCl}_{3}\right): \lambda_{\max }\left(E_{\text {rel }}\right)=458.6(0.22), 490.6(0.60)$, $527.2 \mathrm{~nm}$ (1.00). Fluorescence $\left(\mathrm{CHCl}_{3}\right): \lambda_{\max }\left(I_{\text {rel }}\right)=535.8(1.00), 575.7 \mathrm{~nm}(0.56)$. Fluorescence quantum yield $\left(\mathrm{CHCl}_{3}, \lambda_{\mathrm{exc}}=491 \mathrm{~nm}, E_{491 \mathrm{~nm}, 1 \mathrm{~cm}}=0.0164\right.$, reference: S-13 with $\Phi=1.00):<0.01$. MS (DEP/EI) $m / z(\%): 719(100)\left[M^{+}\right], 538$ (40) $\left[M^{+}+\mathrm{H}-\mathrm{C}_{13} \mathrm{H}_{26}\right], 391(29)\left[M^{+}+\mathrm{H}-\mathrm{C}_{13} \mathrm{H}_{26}-\mathrm{C}_{10} \mathrm{H}_{14} \mathrm{~N}\right]$. HRMS $\left(\mathrm{C}_{47} \mathrm{H}_{49} \mathrm{~N}_{3} \mathrm{O}_{4}\right) \mathrm{m} / \mathrm{z}$ : Calcd. 719.3723, found 719.3729; $\Delta=0.0006 . \mathrm{C}_{47} \mathrm{H}_{49} \mathrm{~N}_{3} \mathrm{O}_{4}$ (719.4): Calcd. C 78.41, H 6.86, N 5.84; found C 78.22, H 6.89, N 5.78. 


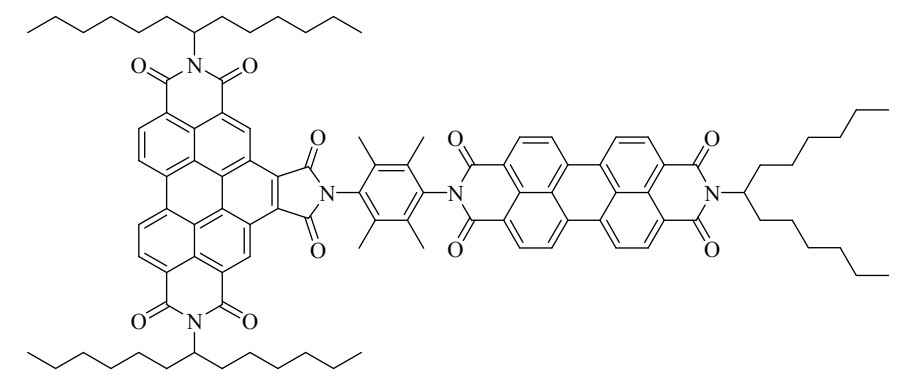

1

2,10-Bis(1-hexylheptyl)-6[4'-(3,8,9,10-tetrahydro-9-(1-hexylheptyl)-1,3,8,10tetraoxoanthra[2,1,9-def:6,5,10-d'e'f']diisoquinoline-2(1H)-yl]2,3,5,6tetramethylphenyl)-1H-pyrrolo[3'4':4,5]pyreno[2,1,10-def:7,8,9d'e'f']diisoquinoline-1,3,5,7,9,11 $(2 \mathrm{H}, 6 \mathrm{H}, 10 H)$-hexone (1):6 2-(4-Amino-2,3,5,6tetramethylphenyl)-9-(1-hexylheptyl)anthra[2,1,9-def;6,5,10-d'e'f']diisoquinoline-1,3,8,10tetraone (RN 1089735-50-1) (150 mg, $208 \mu \mathrm{mol}), 2,10-\mathrm{bis}(1-$ hexylheptyl)furo[3',4':4,5]pyreno[2,1,10-def:7,8,9-d'e'f']diisoquinoline1,3,5,7,9,11(2H,10H)-hexone (RN 130296-37-6) (100 mg, $118 \mu \mathrm{mol})$ and quinoline $\left(3 \mathrm{~mL}\right.$ ) were heated at $230^{\circ} \mathrm{C}$ for $15 \mathrm{~h}$ by means of $250 \mathrm{~W}$ microwave radiation (dark red mixture), allowed to cool, precipitated with vigorous stirring with $2 \mathrm{M}$ aqueous $\mathrm{HCl}$ (200 mL), stirred for $3 \mathrm{~h}$, collected by vacuum filtration (D4 glass filter), washed with $2 \mathrm{~m}$ aqueous $\mathrm{HCl}(300 \mathrm{~mL})$ and distilled water until colorless washings (300 $\mathrm{mL})$, dried at $110^{\circ} \mathrm{C}$ for $2 \mathrm{~d}$ and purified by column separation (silica gel, $\mathrm{CHCl}_{3} /$ methanol $60: 1$ and then neutral alumna, $\mathrm{CHCl}_{3}$ ). Yield $108 \mathrm{mg}(59 \%)$ bright red solid, m.p. $>300^{\circ} \mathrm{C} . R_{\mathrm{f}}$ value $\left(\mathrm{CHCl}_{3} /\right.$ methanol 80:1): 0.7. IR (ATR): $\widetilde{v}=2953$ (m) 2924 (s), 2855 (m), 2361 (w), 2340 (w), 1774 (w), 1721 (s), 1661 (s), 1626 (w), 1594 (m), 1578 (w), 1522 (w), 1484 (w), $1457(\mathrm{~m}), 1431(\mathrm{w}), 1415(\mathrm{w}), 1405(\mathrm{~m}), 1363(\mathrm{~m}), 1336$ (s), 1253 (w), $1204(\mathrm{w})$, ), $1175(w), 1123$ (w), 1018 (w), 963 (w), 851 (w), $810(\mathrm{~m}), 767(\mathrm{w}), 746(\mathrm{~m}), 727(\mathrm{w}), 722$ (w), $667 \mathrm{~cm}^{-1}$ (w). ${ }^{1} \mathrm{H}$ NMR $\left(600 \mathrm{MHz}, \mathrm{CDCl}_{3}, 27.0^{\circ} \mathrm{C}, \mathrm{TMS}\right): \delta=10.62\left(\mathrm{~s}, 1 \mathrm{H}, \mathrm{CH}_{\text {arom }}\right.$.

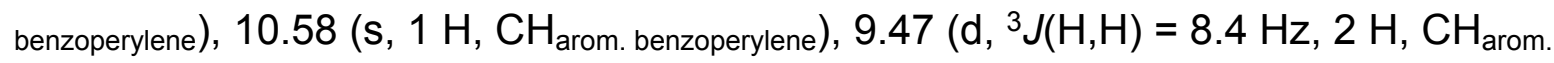

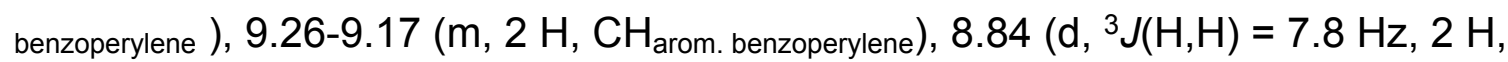

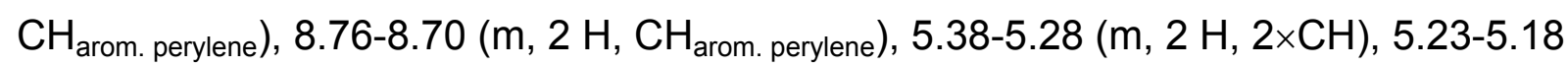
(m, $1 \mathrm{H}, \mathrm{CH}), 2.42-2.31\left(\mathrm{~m}, 4 \mathrm{H}, 2 \times \beta-\mathrm{CH}_{2}\right), 2.30\left(\mathrm{~s}, 6 \mathrm{H}, 2 \times \mathrm{CH}_{3}\right), 2.29-2.22(\mathrm{~m}, 2 \mathrm{H}, \beta-$ $\left.\mathrm{CH}_{2}\right), 2.21\left(\mathrm{~s}, 6 \mathrm{H}, 2 \times \mathrm{CH}_{3}\right), 2.00-1.91\left(\mathrm{~m}, 4 \mathrm{H}, 2 \times \beta-\mathrm{CH}_{2}\right), 1.91-1.85\left(\mathrm{~m}, 2 \mathrm{H}, \beta-\mathrm{CH}_{2}\right)$, 1.46-1.20 (m, $\left.48 \mathrm{H}, 24 \times \mathrm{CH}_{2}\right), 0.85-0.82 \mathrm{ppm}\left(\mathrm{m}, 18 \mathrm{H}, 6 \times \mathrm{CH}_{3}\right) .{ }^{13} \mathrm{C}$ NMR $(150 \mathrm{MHz}$, $\left.\mathrm{CDCl}_{3}, 27.0^{\circ} \mathrm{C}\right): \delta=167.1,162.8,135.4,134.2,132.9,132.5,130.2,128.4,127.5$, 125.3, 124.2, 123.6, 123.4, 123.2, 55.3, 54.8, 32.4, 31.8, 29.7, 29.2, 27.0, 26.9, 22.6, 15.9, 15.4, 14.0 ppm. UV/VIS $\left(\mathrm{CHCl}_{3}\right): \lambda_{\max }(\varepsilon): 376.8$ (41100), 410.6 (16500), 436.0 
(43900), 465.8 (76700), 490.4 (57100), $527.2 \mathrm{~nm}$ (93900). Fluorescence $\left(\mathrm{CHCl}_{3}\right): \lambda_{\max }$ $\left(I_{\text {rel }}\right): 534.8$ (1.00), $577.2 \mathrm{~nm}$ (0.51). Fluorescence quantum yield $\left(\mathrm{CHCl}_{3}, \lambda_{\text {exc }}=490 \mathrm{~nm}\right.$, $E_{490 \mathrm{~nm}, 1 \mathrm{~cm}}=0.0162$, reference: $\mathrm{C} 25$ with $\left.\Phi=1.00\right): 1.00$. Fluorescence quantum yield $\left(\mathrm{CHCl}_{3}, \lambda_{\text {exc }}=436 \mathrm{~nm}, E_{436 \mathrm{~nm}, 1 \mathrm{~cm}}=0.0123\right.$, reference: $\mathrm{C} 25$ with $\left.\Phi=1.00\right): 1.00$.

Fluorescence quantum yield $\left(\mathrm{CHCl}_{3}, \lambda_{\text {exc }}=436 \mathrm{~nm}, E_{436 \mathrm{~nm}, 1 \mathrm{~cm}}=0.0124\right.$, reference: 3,4,9,10-perylenetramethylester with $\Phi=1.00): 1.00$. MS (DEP/EI): $m / z(\%)$ : $1550(14)\left[M^{+}\right], 1368(39)\left[M^{+}+\mathrm{H}-\mathrm{C}_{13} \mathrm{H}_{26}\right], 1186(50)\left[M^{+}+\mathrm{H}-2 \cdot \mathrm{C}_{13} \mathrm{H}_{26}\right], 1004$ (74) $\left[M^{+}+\mathrm{H}-3 \cdot \mathrm{C}_{13} \mathrm{H}_{26}\right] . \mathrm{MS}(\mathrm{FAB}+) \mathrm{m} / \mathrm{z}: 1550\left[M^{+}\right], 1368\left[M^{+}-\mathrm{C}_{13} \mathrm{H}_{26}\right]$, $1186\left[M^{+}-2 \cdot \mathrm{C}_{13} \mathrm{H}_{26}\right], 1004\left[M^{++1}-3 \cdot \mathrm{C}_{13} \mathrm{H}_{26}\right]$. HRMS $\left(\mathrm{C}_{101} \mathrm{H}_{107} \mathrm{~N}_{5} \mathrm{O}_{10}\right) \mathrm{m} / \mathrm{z}:$ Calcd. 1550.8135, found 1550.8051; $\Delta=0.0084 . \mathrm{C}_{101} \mathrm{H}_{107} \mathrm{~N}_{5} \mathrm{O}_{10}$ (1549.8): Calcd. C 78.21, H 6.95, N 4.52; found C 77.84, H 7.03, N 4.35.

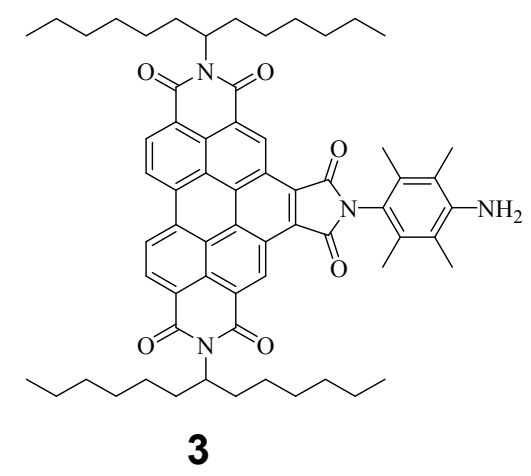

\section{6-(4-Amino-2,3,5,6-tetramethylphenyl)-2,10-bis(1-hexylheptyl)-1H-}

\section{pyrrolo[3'4':4,5]pyreno[2,1,10-def:7,8,9-d'e'f']diisoquinoline-1,3,5,7,9,11}

$\mathbf{( 2 H , 6 H , 1 0 H ) - h e x o n e ~ ( 3 ) : 8 ~ 2 , 3 , 5 , 6 - T e t r a m e t h y l b e n z e n e - 1 , 4 - d i a m i n e ~ ( R N ~ 3 1 0 2 - 8 7 - 2 ) ~}$

(49 mg, $0.30 \mathrm{mmol}), 2,10$-bis(1-hexylheptyl)furo[3',4':4,5]pyreno[2,1,10-def:7,8,9-

d'e'f']diisoquinoline-1,3,5,7,9,11(2H,10H)-hexone (2) (42.5 mg, $50.0 \mu \mathrm{mol})$ and quinoline $\left(2 \mathrm{~mL}\right.$ ) were stirred at $220^{\circ} \mathrm{C}$ and $100 \mathrm{~W}$ microwave radiation for $4 \mathrm{~h}$ (dark yellow mixture), allowed to cool, still warm dropped with vigorous stirring into $2 \mathrm{M}$ aqueous $\mathrm{HCl}$ $(300 \mathrm{~mL})$, stirred for $2 \mathrm{~h}$, allowed to stand for $16 \mathrm{~h}$, collected by vacuum filtration (D4 glass filter), washed with $2 \mathrm{~m}$ aqueous $\mathrm{HCl}(300 \mathrm{~mL})$ and hot distilled water $(300 \mathrm{~mL})$, dried at $110^{\circ} \mathrm{C}$ for $16 \mathrm{~h}$ and purified by column separation (silica gel, $\mathrm{CHCl}_{3} /$ methanol $60: 1)$. Yield $39 \mathrm{mg}(78 \%)$ yellowish green solid, m.p. $>300^{\circ} \mathrm{C} . R_{\mathrm{f}}$ value $\left(\mathrm{CHCl}_{3} / \mathrm{methanol}\right.$ 60:1): 0.7. IR (ATR): $\widetilde{v}=3486(\mathrm{w}), 3404(\mathrm{w}), 3081(\mathrm{w}), 2953(\mathrm{~m}), 2922(\mathrm{~m}), 2854(\mathrm{~m})$, 2358 (w), 1771 (w), 1711 (s), 1660 (s), 1625 (m), 1594 (m), 1522 (w), $1495(\mathrm{~m}), 1415$ (m), $1395(\mathrm{~m}), 1375(\mathrm{~m}), 1365$ (m), 1345 (w), 1309 (s), $1276(\mathrm{w}), 1250$ (w), $1234(\mathrm{w})$, $1206(\mathrm{w}), 1176(\mathrm{w}), 1113(\mathrm{~m}), 945(\mathrm{w}), 875(\mathrm{w}), 848(\mathrm{w}), 813(\mathrm{~m}), 797(\mathrm{w}), 780(\mathrm{w}), 767$ (m), $750(\mathrm{~m}), 725$ (w), $699(\mathrm{w}), 661(\mathrm{w}), 647 \mathrm{~cm}^{-1}(\mathrm{w}) .{ }^{1} \mathrm{H}$ NMR $\left(600 \mathrm{MHz}, \mathrm{CDCl}_{3}\right.$,

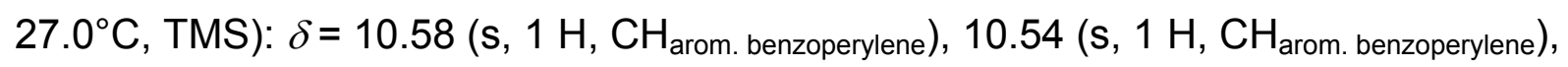


$9.45\left(\mathrm{~d},{ }^{3} \mathrm{~J}(\mathrm{H}, \mathrm{H})=8.4 \mathrm{~Hz}, 2 \mathrm{H}, \mathrm{CH}_{\text {arom. benzoperylene }}\right), 9.26-9.14\left(\mathrm{~m}, 2 \mathrm{H}, \mathrm{CH}_{\text {arom. benzoperylene }}\right)$, 5.36-5.25 (m, $2 \mathrm{H}, 2 \times \mathrm{CH}), 3.90\left(\mathrm{br}, 2 \mathrm{H}, \mathrm{NH}_{2}\right), 2.39-2.28\left(\mathrm{~m}, 4 \mathrm{H}, 2 \times \beta-\mathrm{CH}_{2}\right), 2.22(\mathrm{~s}$, $\left.6 \mathrm{H}, 2 \times \mathrm{CH}_{3}\right), 2.20\left(\mathrm{~s}, 6 \mathrm{H}, 2 \times \mathrm{CH}_{3}\right), 1.97-1.91\left(\mathrm{~m}, 4 \mathrm{H}, 2 \times \beta-\mathrm{CH}_{2}\right), 1.45-1.16(\mathrm{~m}, 32 \mathrm{H}$, $\left.16 \times \mathrm{CH}_{2}\right), 0.82 \mathrm{ppm}\left(\mathrm{t},{ }^{3} \mathrm{~J}(\mathrm{H}, \mathrm{H})=7.0 \mathrm{~Hz}, 12 \mathrm{H}, 4 \times \mathrm{CH}_{3}\right) .{ }^{13} \mathrm{C} \mathrm{NMR}\left(150 \mathrm{MHz}, \mathrm{CDCl}_{3}\right.$, 27.0 ${ }^{\circ} \mathrm{C}$, TMS): $\delta=167.5,133.4,132.5,130.1,128.3,127.8,127.5,125.3,124.1,123.6$, 55.3, 32.4, 31.8, 29.2, 27.0, 22.6, 15.5, 14.0 ppm. UV/VIS $\left(\mathrm{CHCl}_{3}\right): \lambda_{\max }(\varepsilon)=273.4$ (35000), 371.8 (37400), 411.2 (14900), 436.0 (39100), $465.8 \mathrm{~nm}$ (60300). Fluorescence quantum yield $\left(\mathrm{CHCl}_{3}, \lambda_{\text {exc }}=436 \mathrm{~nm}, E_{436 \mathrm{~nm}, 1 \mathrm{~cm}}=0.0131\right.$, reference: $\mathrm{C} 25$ with $\Phi=$ 1.00): $<0.01$. MS (DEP/EI) $m / z(\%): 995$ (100) $\left[M^{+}+\mathrm{H}\right], 813(28)\left[M^{+}+\mathrm{H}-\mathrm{C}_{13} \mathrm{H}_{26}\right]$, 631 (19) $\left[M^{+}+\mathrm{H}-2 \cdot \mathrm{C}_{13} \mathrm{H}_{26}\right]$. HRMS $\left(\mathrm{C}_{64} \mathrm{H}_{75} \mathrm{~N}_{4} \mathrm{O}_{6}\right) \mathrm{m} / \mathrm{z}$ : Calcd. 995.5608, found 995.5656; $\Delta=0.0048 . \mathrm{C}_{64} \mathrm{H}_{74} \mathrm{~N}_{4} \mathrm{O}_{6}$ (994.6): Calcd. C 77.23, H 7.49, N 5.63; found C 77.08, H 7.46, N 5.53 .

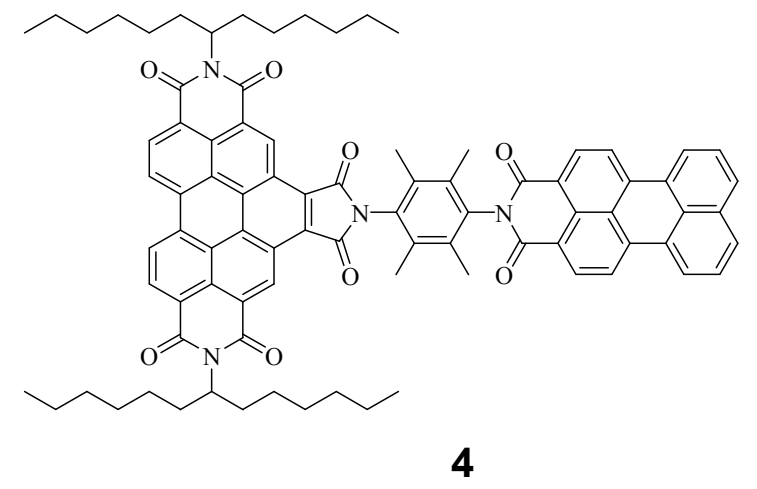

2,10-Bis(1-hexylheptyl)-[6-(4'-(benzo[5,10]anthra[2,1,9-def]isoquinoline-1,3(2H)dione)]-2,3,5,6-tetramethylphenyl-1H-pyrrolo[3'4':4,5]pyreno[2,1,10-def:7,8,9d'e'f']diisoquinoline-1,3,5,7,9,11 (2H,6H,10H)-hexone (4): 6-(4-Amino-2,3,5,6tetramethylphenyl)-2,10-bis(1-hexylheptyl)-1H-pyrrolo[3'4':4,5]pyreno[2,1,10-def:7,8,9d'e'f']diisoquinoline-1,3,5,7,9,11(2H,6H,10H)-hexone (3) (150 mg, $151 \mu \mathrm{mol})$, benzo[5,10]anthra[2,1,9-def]isochromen-1,3-dione 4 (40 mg, $0.12 \mathrm{mmol}$ ), zincacetate (5 mg, $27 \mu \mathrm{mol})$ and quinoline $(3 \mathrm{~mL})$ were stirred at $210^{\circ} \mathrm{C}$ for $8.5 \mathrm{~h}$ (orange mixture), allowed to cool, still warm dropped with vigorous stirring into $2 \mathrm{~m}$ aqueous $\mathrm{HCl}(400 \mathrm{~mL})$, stirred for $2 \mathrm{~h}$, allowed to stand for $16 \mathrm{~h}$, collected by vacuum filtration (D4 glass filter), washed with $2 \mathrm{~m}$ aqueous $\mathrm{HCl}(500 \mathrm{~mL})$, hot distilled water $(500 \mathrm{~mL})$ and a mixture of methanol and distilled water $(500 \mathrm{~mL}, 1: 1)$, dried at $110^{\circ} \mathrm{C}$ for $2 \mathrm{~d}$ and purified by column separation (neutral alumina, $\mathrm{CHCl}_{3} /$ methanol $80: 1$ and then silica gel, $\mathrm{CHCl}_{3} /$ methanol 60:1). Yield $35 \mathrm{mg}(21 \%)$ red solid, m.p. $>300^{\circ} \mathrm{C} . R_{\mathrm{f}}$ value $\left(\mathrm{CHCl}_{3} / \mathrm{MeOH}=60: 1\right): 0.8$. IR (ATR): $\widetilde{v}=3853(\mathrm{w}), 3744(\mathrm{w}), 3649(\mathrm{w}), 3322(\mathrm{w}), 2922(\mathrm{~m}), 2856(\mathrm{~m}), 2361(\mathrm{w})$, 2339 (w), 1973 (w), 1925 (w), $1772(w), 1718(\mathrm{~m}), 1704$ (s), $1666(\mathrm{~s}), 1625$ (w), 1593.0 (m), $1578(w), 1521(w), 1500(w), 1457(m), 1413(m), 1393(w), 1352(s), 1317(s)$, 
$1273(w), 1246(m), 1233(w), 1198(w), 1176(m), 1114(w), 1112(w), 1021(w), 971$ (w), 945 (w), 918 (w), 885 (w), 854 (w), 845 (w), 837 (w), 810 (s), 795 (w), $761(\mathrm{~m}), 753$ (m), 747 (s), 726 (w), 698 (w), 665 (w), 659 (w), $644(w), 630(w), 621(w), 613 \mathrm{~cm}^{-1}(\mathrm{w})$. ${ }^{1} \mathrm{H}$ NMR $\left(600 \mathrm{MHz}, \mathrm{CDCl}_{3}, 27.0^{\circ} \mathrm{C}, \mathrm{TMS}\right): \delta=10.61\left(\mathrm{~s}, 1 \mathrm{H}, \mathrm{CH}_{\text {arom. benzoperylene }}\right), 10.56$

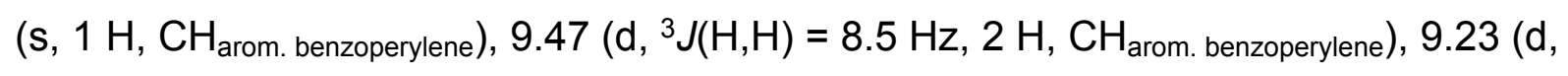
$\left.{ }^{3} \mathrm{~J}(\mathrm{H}, \mathrm{H})=8.4 \mathrm{~Hz}, 2 \mathrm{H}, \mathrm{CH}_{\text {arom. benzoperylene }}\right), 8.72\left(\mathrm{~d},{ }^{3} \mathrm{~J}(\mathrm{H}, \mathrm{H})=7.8 \mathrm{~Hz}, 2 \mathrm{H}, \mathrm{CH}_{\text {arom. perylene }}\right)$, $8.50\left(\mathrm{~d},{ }^{3} \mathrm{~J}(\mathrm{H}, \mathrm{H})=7.8 \mathrm{~Hz}, 2 \mathrm{H}, \mathrm{CH}_{\text {arom. perylene }}\right), 8.49\left(\mathrm{~d},{ }^{3} \mathrm{~J}(\mathrm{H}, \mathrm{H})=7.8 \mathrm{~Hz}, 2 \mathrm{H}, \mathrm{CH}_{\text {arom. }}\right.$

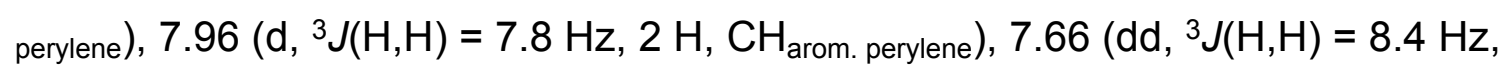

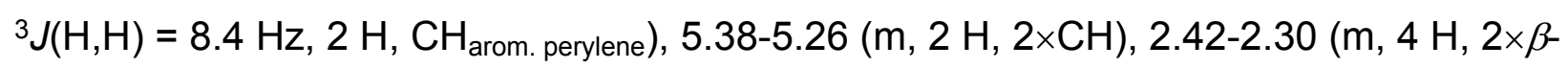
$\left.\mathrm{CH}_{2}\right), 2.28\left(\mathrm{~s}, 6 \mathrm{H}, 2 \times \mathrm{CH}_{3}\right), 2.20\left(\mathrm{~s}, 6 \mathrm{H}, 2 \times \mathrm{CH}_{3}\right), 1.99-1.90\left(\mathrm{~m}, 4 \mathrm{H}, 2 \times \beta-\mathrm{CH}_{2}\right), 1.46-$ $1.17\left(\mathrm{~m}, 32 \mathrm{H}, 16 \times \mathrm{CH}_{2}\right), 0.82 \mathrm{ppm}\left(\mathrm{t},{ }^{3} \mathrm{~J}(\mathrm{H}, \mathrm{H})=7.0 \mathrm{~Hz}, 12 \mathrm{H}, 4 \times \mathrm{CH}_{3}\right) .{ }^{13} \mathrm{C} \mathrm{NMR}$ $\left(150 \mathrm{MHz}, \mathrm{CDCl}_{3}, 27.0^{\circ} \mathrm{C}, \mathrm{TMS}\right): \delta=167.1,163.3,137.8,133.9,133.5,133.0,132.1$, 131.1, 129.2, 128.4, 127.6, 127.1, 125.3, 124.1, 124.0, 123.6, 120.9, 120.3, 55.3, 32.4, $31.8,29.7,29.2,27.0,22.6,15.9,15.4,14.0 \mathrm{ppm}$. UVIVIS $\left(\mathrm{CHCl}_{3}\right): \lambda_{\max }\left(E_{\mathrm{rel}}\right)=264.6$ (0.69), 374.6 (0.46), 410.6 (0.21), 436.8 (0.57), 466.6 (1.00), 511.2 nm (0.56).

Fluorescence $\left(\mathrm{CHCl}_{3}\right): \lambda_{\max }\left(I_{\text {rel }}\right)=544.9$ (1.00), $582.9 \mathrm{~nm}(0.80)$. Fluorescence quantum yield $\left(\mathrm{CHCl}_{3}, \lambda_{\text {exc }}=436 \mathrm{~nm}, E_{436 \mathrm{~nm}, 1 \mathrm{~cm}}=0.0214\right.$, reference: $3,4,9,10$ Perylenetramethylester with $\Phi=1.00)$ : 0.68. MS (FAB) $m / z: 1299\left[M^{+}+\mathrm{H}\right], 935\left[M^{+}+\mathrm{H}-\right.$ 2. $\mathrm{C}_{13} \mathrm{H}_{26}$ ]. $\mathrm{C}_{76} \mathrm{H}_{78} \mathrm{~N}_{4} \mathrm{O}_{8}$ (1298.6): Calcd. C 79.48, H 6.36, N 4.31; found C 78.65, H 6.14, N 4.36 .

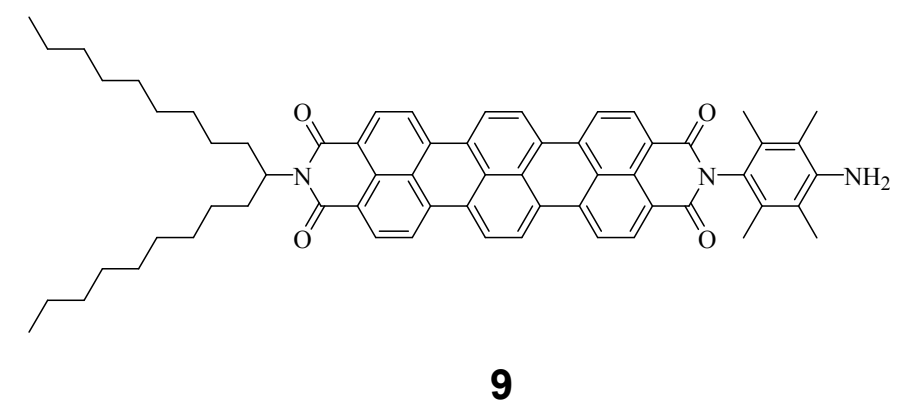

\section{2-(4-Amino-2,3,5,6-tetramethylphenyl)-11-(1-nonyldecyl)-} benzo[13,14]pentapheno[3,4,5-def:10,9,8-d'e'f']diisoquinoline-1,3,10,12(2H,11H)tetraone (9): 2,3,5,6-Tetramethylbenzene-1,4-diamine (RN 3102-87-2) (66 mg, $0.40 \mathrm{mmol}), 11-($ nonyldecyl)-1Hbenzo[13,14]isochromeno[6',5',4':8,9,10]pentapheno[3,4,5-def]isoquinoline1,3,10,12(11H)-tetraone (8) (78 mg, $0.10 \mathrm{mmol})$, zincacetate (11 mg, $60 \mu \mathrm{mol})$ and quinoline $\left(1.8 \mathrm{~mL}\right.$ ) under argon atmosphere were stirred at $210^{\circ} \mathrm{C}$ for $4 \mathrm{~h}$ (deep blue mixture), allowed to cool, still warm dropped with vigorous stirring into $2 \mathrm{~m}$ aqueous $\mathrm{HCl}$ $(300 \mathrm{~mL})$, stirred for $2 \mathrm{~h}$, allowed to stand for $16 \mathrm{~h}$, collected by vacuum filtration (D4 
glass filter), washed with $2 \mathrm{~m}$ aqueous $\mathrm{HCl}(300 \mathrm{~mL})$, hot distilled water $(300 \mathrm{~mL})$ and a mixture of methanol and distilled water $(300 \mathrm{~mL}, 1: 1)$, dried in vacuo at $110^{\circ} \mathrm{C}$ for $2 \mathrm{~d}$ and purified by column separation (neutral alumina, $\mathrm{CHCl}_{3} /$ methanol $80: 1$ and then silica gel, $\mathrm{CHCl}_{3} /$ methanol 40:1). Yield $71 \mathrm{mg}(76 \%)$ deep blue solid, m.p. $>300^{\circ} \mathrm{C}, R_{\mathrm{f}}$ value (silica gel, $\mathrm{CHCl}_{3} /$ methanol 40:1):0.4. IR (ATR): $\widetilde{v}=3482(\mathrm{w}), 3398(\mathrm{w}), 2920(\mathrm{~m}), 2850$ (m), 1693 (s), 1653 (s), 1632 (s), 1584 (s), 1506 (w), 1467 (m), 1437 (m), 1418 (w), $1379(\mathrm{~m}), 1351$ (s), 1322 (s), 1302 (s), 1250 (m), 1206 (m), 1182 (w), 1139 (w), 1119 (m), $1053(\mathrm{w}), 1009(\mathrm{w}), 854(\mathrm{w}), 841(\mathrm{~m}), 806(\mathrm{~s}), 790(\mathrm{~m}), 750(\mathrm{~m}), 721(\mathrm{~m}), 694(\mathrm{w})$, $680(\mathrm{w}), 668 \mathrm{~cm}^{-1}(\mathrm{w}) .{ }^{1} \mathrm{H} \mathrm{NMR}\left(600 \mathrm{MHz}^{\mathrm{C}} \mathrm{CDCl}_{3}, 27.0^{\circ} \mathrm{C}, \mathrm{TMS}\right): \delta=8.70\left(\mathrm{~d},{ }^{3} \mathrm{~J}(\mathrm{H}, \mathrm{H})=\right.$

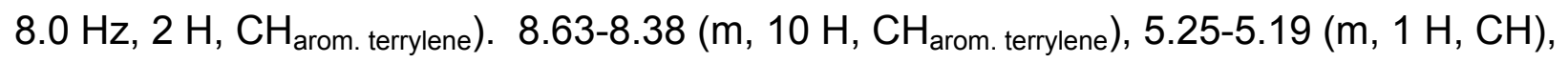
$3.73\left(\mathrm{br}, 2 \mathrm{H}, \mathrm{NH}_{2}\right), 2.32-2.24\left(\mathrm{~m}, 2 \mathrm{H}, \beta-\mathrm{CH}_{2}\right), 2.17\left(\mathrm{~s}, 6 \mathrm{H}, 2 \times \mathrm{CH}_{3}\right), 2.10(\mathrm{~s}, 6 \mathrm{H}$, $\left.2 \times \mathrm{CH}_{3}\right), 1.93-1.86\left(\mathrm{~m}, 2 \mathrm{H}, \beta-\mathrm{CH}_{2}\right), 1.42-1.13\left(\mathrm{~m}, 28 \mathrm{H}, 14 \times \mathrm{CH}_{2}\right), 0.83 \mathrm{ppm}(\mathrm{t}, 3 \mathrm{~J}(\mathrm{H}, \mathrm{H})=$ 7.1 Hz, $\left.6 \mathrm{H}, 2 \times \mathrm{CH}_{3}\right) .{ }^{13} \mathrm{C} \mathrm{NMR}\left(150 \mathrm{MHz}, \mathrm{CDCl}_{3}, 27.0^{\circ} \mathrm{C}\right): \delta=163.7,135.9,135.3$, $131.9,131.0,130.9,130.8,130.3,129.7,128.5,126.2,125.8,124.2,124.1,121.9$, 121.3, 118.9, 54.6, 32.4, 31.9, 29.7, 29.6, 29.3, 27.0, 22.6, 15.1, 14.1, 13.9 ppm. UVIVIS $\left(\mathrm{CHCl}_{3}\right): \lambda_{\max }(\varepsilon)=555.3$ (22300), 600.4 (66300), $653.8 \mathrm{~nm}$ (130400).

Fluorescence $\left(\mathrm{CHCl}_{3}\right): \lambda_{\max }\left(I_{\text {rel }}\right)=673.9$ (1.00), $731.3 \mathrm{~nm}(0.43)$. Fluorescence quantum yield $\left(\mathrm{CHCl}_{3}, \Lambda_{\mathrm{exc}}=600 \mathrm{~nm}, E_{600 \mathrm{~nm}, 1 \mathrm{~cm}}=0.0322\right.$, reference: $\mathrm{S}$-19Terrylene with $\Phi=$

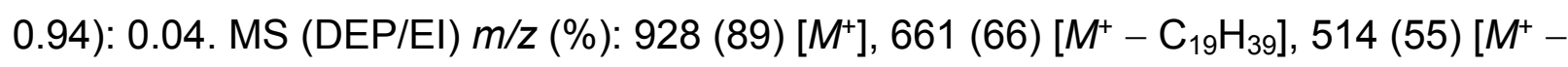
$\left.\mathrm{C}_{19} \mathrm{H}_{39}-\mathrm{C}_{10} \mathrm{H}_{15} \mathrm{~N}\right]$. HRMS $\left(\mathrm{C}_{63} \mathrm{H}_{65} \mathrm{~N}_{3} \mathrm{O}_{4}\right) \mathrm{m} / \mathrm{z}$ : Calcd. 927.4975, found 927.4984; $\Delta=$ 0.0009. $\mathrm{C}_{63} \mathrm{H}_{65} \mathrm{~N}_{3} \mathrm{O}_{4}$ (927.5): Calcd. C 81.52, H 7.06, N 4.53; found C 80.70, H 7.09, N 4.33 .

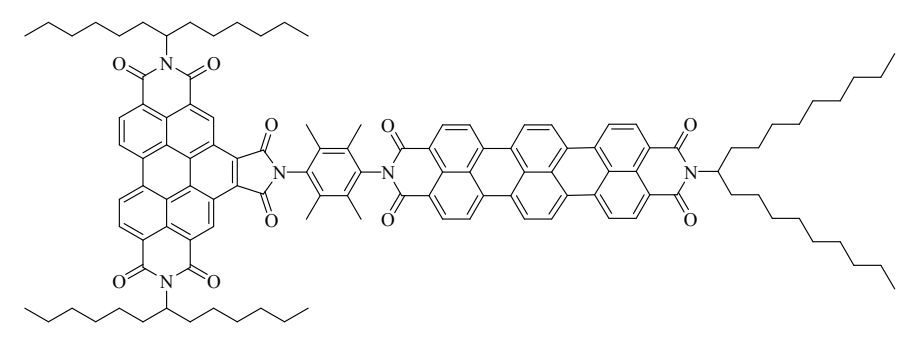

10

\section{2,10-Bis(1-hexylheptyl)-6[4'-(3,8,9,10-tetrahydro-11-(1-} nonyldecyl)benzo[13,14]pentapheno1,3,8,10-tetraoxoanthra[3,4,5-def:10,9,8d'e'f']diisoquinoline-1,3,10,12(2H,11H)-yl-2,3,5,6-tetramethylphenyl)-1Hpyrrolo[3'4':4,5]pyreno[2,1,10-def:7,8,9-d'e'f']diisoquinoline1,3,5,7,9,11(2H,6H,10H)-hexone (10): 2-(4-Amino-2,3,5,6-tetramethyl-phenyl)-11-(1nonyldecyl)-benzo[13,14]pentapheno[3,4,5-def:10,9,8-d'e'f']diisoquinoline1,3,10,12(2H,11H)-tetraone (9) $(28 \mathrm{mg}, 30 \mu \mathrm{mol}), 2,10$-bis(1- 
hexylheptyl)furo[3',4':4,5]pyreno[2,1,10-def:7,8,9-d'e'f]ddiisoquinoline-

1,3,5,7,9,11(2H,10H)-hexaone (2) (51 mg, $60 \mu \mathrm{mol})$ and quinoline $(2 \mathrm{~mL})$ were heated at $230^{\circ} \mathrm{C}$ and $200 \mathrm{~W}$ microwave radiation for $9 \mathrm{~h}$ (greenish blue mixture), allowed to cool, still warm dropped with vigorous stirring into $2 \mathrm{~m}$ aqueous $\mathrm{HCl}(300 \mathrm{~mL})$, stirred for $2 \mathrm{~h}$, allowed to stand for $16 \mathrm{~h}$, collected by vacuum filtration (D4 glass filter), washed with $2 \mathrm{~m}$ aqueous $\mathrm{HCl}(300 \mathrm{~mL})$, hot distilled water $(300 \mathrm{~mL})$ and a mixture of methanol and distilled water $(300 \mathrm{~mL}, 1: 1)$, dried in vacuo at $110^{\circ} \mathrm{C}$ for $18 \mathrm{~h}$ and purified by column separation (basic alumina, $\mathrm{CHCl}_{3} /$ methanol $80: 1$; silica gel, $\mathrm{CHCl}_{3} /$ methanol 80:1; neutral alumina, dichloromethane; weakly acid alumina, $\mathrm{CHCl}_{3} /$ methanol 100:1). Yield $34 \mathrm{mg}(64 \%)$ dark green solid, m.p. $>300^{\circ} \mathrm{C}$. $R_{\mathrm{f}}$ value $\left(\mathrm{CHCl}_{3} /\right.$ methanol $\left.60: 1\right): 0.8$. IR (ATR): $\widetilde{v}=2957$ (m) 2924 (m), 2855 (m), $1774(\mathrm{w}), 1721(\mathrm{~m}), 1707$ (s), $1662(\mathrm{~s})$, $1587(\mathrm{~s}), 1522(\mathrm{w}), 1506(\mathrm{w}), 1460(\mathrm{~m}), 1415(\mathrm{~m}), 1395(\mathrm{w}), 1379(\mathrm{~m}), 1354(\mathrm{~s}), 1319$ (s), $1260(\mathrm{~m}), 1204(\mathrm{w}), 1176(\mathrm{w}), 1095(\mathrm{~m}), 1017(\mathrm{w}), 946(\mathrm{w}), 869(\mathrm{w}), 842(\mathrm{w}), 809$ (s), $767(w), 749(\mathrm{~m}), 723(\mathrm{w}), 696(\mathrm{w}), 684(\mathrm{w}), 662(\mathrm{w}), 645 \mathrm{~cm}^{-1}(\mathrm{w}) .{ }^{1} \mathrm{H}$ NMR $(600$ $\left.\mathrm{MHz}, \mathrm{CDCl}_{3}, 27.0^{\circ} \mathrm{C}, \mathrm{TMS}\right): \delta=10.66-10.58\left(\mathrm{~m}, 2 \mathrm{H}, \mathrm{CH}_{\text {arom. benzoperylene }}\right), 9.51$ (d, $\left.{ }^{3} \mathrm{~J}(\mathrm{H}, \mathrm{H})=8.5 \mathrm{~Hz}, 2 \mathrm{H}, \mathrm{CH}_{\text {arom. benzoperylene }}\right)$, 9.29-9.19 ( $\left.\mathrm{m}, 2 \mathrm{H}, \mathrm{CH}_{\text {arom. benzoperylene }}\right)$, 8.84$8.58\left(\mathrm{~m}, 10 \mathrm{H}, \mathrm{CH}_{\text {arom. terrylene }}\right), 5.39-5.28\left(\mathrm{~m}, 2 \mathrm{H}, \mathrm{CH}_{\text {benzoperylene }}\right), 5.26-5.19(\mathrm{~m}, 1 \mathrm{H}$,

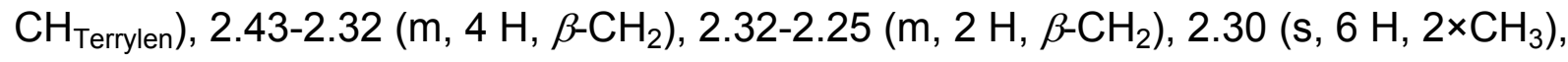
$2.22\left(\mathrm{~s}, 6 \mathrm{H}, 2 \times \mathrm{CH}_{3}\right), 2.00-1.92\left(\mathrm{~m}, 4 \mathrm{H}, \beta-\mathrm{CH}_{2}\right), 1.92-1.84\left(\mathrm{~m}, 2 \mathrm{H}, \beta-\mathrm{CH}_{2}\right), 1.46-1.17$ $\left(\mathrm{m}, 60 \mathrm{H}, 30 \times \mathrm{CH}_{2}\right), 0.85-0.80 \mathrm{ppm}\left(\mathrm{m}, 18 \mathrm{H}, 6 \times \mathrm{CH}_{3}\right) .{ }^{13} \mathrm{C} \mathrm{NMR}\left(150 \mathrm{MHz}, \mathrm{CDCl}_{3}\right.$, $\left.27.0^{\circ} \mathrm{C}\right): \delta=167.0,163.1,134.0,133.0,130.9,128.4,127.6,123.7,121.7,121.6,55.7$, $55.3,32.4,31.9,31.8,29.6,29.3,27.0,22.6,22.6,15.9,15.4,14.1,14.0$ ppm. UV/VIS $\left(\mathrm{CHCl}_{3}\right): \lambda_{\max }(\varepsilon): 377.1$ (44400), 410.2 (18400), 436.5 (40000), 466.5 (62300), 557.6 (23600), 601.1 (73000), $654.5 \mathrm{~nm}$ (143000). Fluorescence $\left(\mathrm{CHCl}_{3}\right): \lambda_{\max }\left(I_{\text {rel }}\right): 669.9$ (1.00), $734.7 \mathrm{~nm}(0.50)$. Fluorescence quantum yield $\left(\mathrm{CHCl}_{3}, \lambda_{\text {exc }}=437 \mathrm{~nm}, E_{437 \mathrm{~nm}, 1 \mathrm{~cm}}\right.$ $=0.0169$, reference: $\mathrm{C} 25$ with $\Phi=1.00): 0.63$. Fluorescence quantum yield $\left(\mathrm{CHCl}_{3}, \lambda_{\text {exc }}\right.$ $=437 \mathrm{~nm}, E_{437 \mathrm{~nm}, 1 \mathrm{~cm}}=0.0169$, reference: 3,4,9,10-Perylenetetramethylester with $\Phi=$ 1.00): 0.62. Fluorescence quantum yield $\left(\mathrm{CHCl}_{3}, \lambda_{\mathrm{exc}}=601 \mathrm{~nm}, E_{601 \mathrm{~nm}, 1 \mathrm{~cm}}=0.0306\right.$, Reference: perylene-terrylene -dyad with $\Phi=0.89$ ): 0.91. MS (MALDI): (Matrix: Anthracene) $\mathrm{m} / \mathrm{z}: 1758\left[M^{+}\right]$. MS (FAB+) m/z: $1758\left[M^{+}\right], 1576\left[M^{+}-\mathrm{C}_{13} \mathrm{H}_{26}\right], 1492\left[M^{+}-\right.$ $\mathrm{C}_{19} \mathrm{H}_{38}$ ]. HRMS $\left(\mathrm{C}_{117} \mathrm{H}_{123} \mathrm{~N}_{5} \mathrm{O}_{10}\right) \mathrm{m} / \mathrm{z}$ : Calcd. 1758.9304, found 1758.9266; $\Delta=-0.0038$. $\mathrm{C}_{117} \mathrm{H}_{123} \mathrm{~N}_{5} \mathrm{O}_{10}$ (1757.9): Calcd. C 79.88, H 7.05, N 3.98; found C 79.53, H 7.02, N 3.95. 


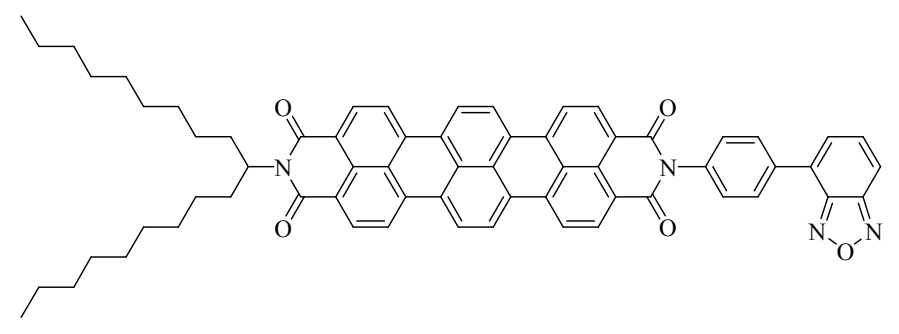

12

2-(4-2,1,3-Benzoxazophenyl)-11-(1-nonyldecyl)-benzo[13,14]pentapheno[3,4,5def:10,9,8-d'e'f]'diisoquinoline-1,3,10,12(2H,11H)-tetraone (12): 4-

Benzo[1,2,5]oxadiazol-4-yl-phenylamine ${ }^{9}$ (11) (37 mg, 0.17 mmol), 11 -(nonadecan-10yl)-1H-benzo[13,14]isochromeno[6',5',4':8,9,10]pentapheno[3,4,5-def]isoquinoline1,3,10,12(11H)tetraone (8) (68 mg, $87 \mu \mathrm{mol})$, zincacetate $(3.2 \mathrm{mg}, 17 \mu \mathrm{mol})$ and quinoline $\left(2.0 \mathrm{~mL}\right.$ ) under argon atmosphere were stirred at $200^{\circ} \mathrm{C}$ for $6 \mathrm{~h}$ (deep blue mixture), allowed to cool, still warm dropped with vigorous stirring into $2 \mathrm{~m}$ aqueous $\mathrm{HCl}$ (300 mL), stirred for $2 \mathrm{~h}$, allowed to stand for $16 \mathrm{~h}$, collected by vacuum filtration (D4 glass filter), washed with $2 \mathrm{M}$ aqueous $\mathrm{HCl}(300 \mathrm{~mL})$, hot distilled water $(300 \mathrm{~mL})$ and a mixture of methanol and distilled water $(300 \mathrm{~mL}, 1: 1)$, dried in vacuo at $90^{\circ} \mathrm{C}$ for $1 \mathrm{~d}$ and purified by column separation (neutral alumina, $\mathrm{CHCl}_{3} /$ methanol $80: 1$ and then silica gel, $\mathrm{CHCl}_{3} /$ methanol $\left.80: 1\right)$. Yield $31 \mathrm{mg}(37 \%)$ blue solid, m.p. $>300^{\circ} \mathrm{C}$. $R_{\mathrm{f}}$ value $\left(\mathrm{CHCl}_{3} /\right.$ methanol 60:1): 0.6. IR (ATR): $\widetilde{v}=2955(\mathrm{w}), 2921(\mathrm{~m}), 2852(\mathrm{~m}), 1691(\mathrm{~s})$, 1652 (s), $1586(\mathrm{~s}), 1506(\mathrm{~m}), 1465(\mathrm{w}), 1421(\mathrm{w}), 1379(\mathrm{~m}), 1355(\mathrm{~s}), 1329(\mathrm{~m}), 1305$ $(\mathrm{m}), 1255(\mathrm{~m}), 1208(\mathrm{~m}), 1184(\mathrm{~m}), 1144(\mathrm{w}), 1114(\mathrm{w}), 1071(\mathrm{w}), 1015(\mathrm{~m}), 965(\mathrm{w})$, $888(w), 856(w), 846(m), 823(m), 808(s), 798(s), 762(w), 746(s), 720(w), 694(w)$, $678(\mathrm{~m}), 648(\mathrm{w}), 616(\mathrm{w}), 608 \mathrm{~cm}^{-1}(\mathrm{w}) .{ }^{1} \mathrm{H} \mathrm{NMR}\left(400 \mathrm{MHz}, \mathrm{C}_{2} \mathrm{D}_{2} \mathrm{Cl}_{4}, 105^{\circ} \mathrm{C}, \mathrm{TMS}\right): \delta=$

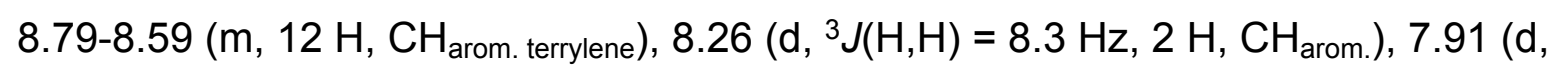
$\left.{ }^{3} \mathrm{~J}(\mathrm{H}, \mathrm{H})=9.0 \mathrm{~Hz}, 1 \mathrm{H}, \mathrm{CH}_{\text {arom. }}\right), 7.74\left(\mathrm{~d},{ }^{3} \mathrm{~J}(\mathrm{H}, \mathrm{H})=6.8 \mathrm{~Hz}, 1 \mathrm{H}, \mathrm{CH}_{\text {arom. }}\right), 7.63-7.58(\mathrm{~m}$, $\left.3 \mathrm{H}, \mathrm{CH}_{\text {arom. }}\right), 5.27-5.19(\mathrm{~m}, 1 \mathrm{H}, \mathrm{CH}), 2.35-2.25\left(\mathrm{~m}, 2 \mathrm{H}, \beta-\mathrm{CH}_{2}\right), 2.02-1.92(\mathrm{~m}, 2 \mathrm{H}, \beta-$ $\left.\mathrm{CH}_{2}\right), 1.47-1.24\left(\mathrm{~m}, 28 \mathrm{H}, 14 \times \mathrm{CH}_{2}\right), 0.91 \mathrm{ppm}\left(\mathrm{t},{ }^{3} \mathrm{~J}(\mathrm{H}, \mathrm{H})=6.8 \mathrm{~Hz}, 6 \mathrm{H}, 2 \times \mathrm{CH}_{3}\right)$. UV/VIS $\left(\mathrm{CHCl}_{3}\right): \lambda_{\max }(\varepsilon)=314.8$ (17400), 341.2 (15900), 558.3 (23000), 601.1 (70300), $655.3 \mathrm{~nm}(139500)$. Fluorescence $\left(\mathrm{CHCl}_{3}\right): \lambda_{\max }\left(I_{\text {rel }}\right)=671.8$ (1.00), $738.0 \mathrm{~nm}(0.50)$. Fluorescence quantum yield $\left(\mathrm{CHCl}_{3}, \lambda_{\text {exc }}=602 \mathrm{~nm}, E_{602 \mathrm{~nm}, 1 \mathrm{~cm}}=0.0128\right.$, reference: S19Terrylene with $\Phi=0.94): 0.90$. Fluorescence quantum yield $\left(\mathrm{CHCl}_{3}, \lambda_{\text {exc }}=350 \mathrm{~nm}\right.$, $E_{350 \mathrm{~nm}, 1 \mathrm{~cm}}=0.0050$, reference: $\mathrm{C} 25$ with $\left.\Phi=1.00\right): 0.67$. Fluorescence lifetime $\left(\mathrm{CHCl}_{3}, \lambda_{\mathrm{exc}}=633 \mathrm{~nm}\right): 3.98 \mathrm{~ns}$. Fluorescence lifetime $\left(\mathrm{CHCl}_{3}, \lambda_{\mathrm{exc}}=341 \mathrm{~nm}\right): 2.74 \mathrm{~ns}$. MS (DEP/EI) m/z (\%): 975 (31) [ $\left.M^{+}+\mathrm{H}\right], 708(94)\left[M^{+}-\mathrm{C}_{19} \mathrm{H}_{38}\right]$. HRMS $\left(\mathrm{C}_{65} \mathrm{H}_{59} \mathrm{~N}_{4} \mathrm{O}_{5}\right) \mathrm{m} / \mathrm{z}$ : 
Calcd. 975.4441, found 975.4490; $\Delta=0.0049 . \mathrm{C}_{65} \mathrm{H}_{58} \mathrm{~N}_{4} \mathrm{O}_{5}$ (974.4): Calcd. C 80.06, H 5.99, N 5.75; found C 79.62, H 5.96, N 5.61.

\subsection{Methods}

All reagents were used as received from commercial sup-pliers. The solvents used in the reactions were dried with standard drying agents and freshly distilled prior to use. Reaction progress was monitored by thin-layer chroma-tography (TLC) on E. Merck Kieselgel 60 F254. Coumn chromatography was performed using silica gel (60 A, 40-63 $\mu \mathrm{m}, \mathrm{ROCC})$. IR spectra were recorded as ATR with a Perkin Elmer 1420 Ratio Recording Infrared Spektrometer, FT 1000 (4000-450 cm-1). UV/Vis/NIR spectra: Varian Cary 5000; fluorescence spectra: Varian Carry (totally corrected). All ${ }^{1} \mathrm{H}$ and ${ }^{13} \mathrm{C}$ NMR spectra were recorded with a Varian Vnmrs $600(600 \mathrm{MHz})$ in $\mathrm{CDCl}_{3}$. Chemical shifts $(\delta)$ are reported in ppm and coupling constants $(J)$ in $\mathrm{Hz}$. Mass spectra were performed using a Finnigan MAT 95. Elemental analyses $(\mathrm{C}, \mathrm{H}, \mathrm{N})$ were conducted using the Elemental Analyser Elementar Vario EL from Elementar Analysensysteme $\mathrm{GmbH}$. Fluorescence lifetimes $\tau$ were obtained with a PicoQuant 300lifetime spectrometer and a PicoQuant, P-C-405 as a light source.

Overlap spectra and overlap integrals were exactly calculated as was described in ref. ${ }^{10}$ (page 122) where the higher resolution of the spectra of $0.2 \mathrm{~nm}$ was considered. Transient spectra were exactly recorded as is described in ref. ${ }^{11}$

\section{References}

1. H. Langhals, J. Karolin and L. B.-Å. Johansson, 'Spectroscopic properties of new and convenient standards for measuring fluorescence quantum yields', J. Chem. Soc., Faraday Trans., 1998, 94, 2919-2922.

2. S. Kalinin, M. Speckbacher, H. Langhals and L. B.-Å. Johansson, 'A new and versatile fluorescence standard for quantum yield determination', Phys. Chem. Chem. Phys., 2001, 3, 172-174.

3. H. Langhals, A. Walter, E. Rosenbaum and L. B.-Á. Johansson, 'A versatile standard for bathochromic fluorescence based on intramolecular FRET', Phys. Chem. Chem. Phys., 2011, 13, 11055-11059.

4. L. Feiler, H. Langhals and K. Polborn, 'Synthesis of perylene-3,4-dicarboximides Novel, highly photostable fluorescent dyes', Liebigs Ann. Chem., 1995, 1229-1244.

5. H. Kaiser, J. Lindner and H. Langhals, 'Synthese von nichtsymmetrisch substituierten Perylen-Fluoreszenzfarbstoffen', Chem. Ber., 1991, 124, 529-535. 
6. H. Langhals, S. Poxleitner, O. Krotz, T. Pust and A. Walter, 'FRET in orthogonally arranged chromophores', Eur. J. Org. Chem., 2008, 4559-4562.

7. L. I. Smith, 'Duroquinone', Organic Syntheses, 1930, 10, 40-42.

8. S. Zimdars, H. Langhals, P. Knochel, 'Functionalization of the benzo[c][1,2,5]thiadiazole scaffold via Mg-, Zn- and Mn-intermediates', Synthesis 2011, 1302-1308.

9. H. Langhals and A. Hofer, 'Chromophores arranged as 'magnetic meta atoms': Building blocks for molecular metamaterials', J. Org. Chem., 2013, 78, 5889-5897. 10. N. Hildebrandt, How to apply FRET: from experimental design to data analysis, in I. Medintz (ed.), FRET - Förster resonance energy transfer. From theory to applications, Wiley VCH, Weinheim 2014; ISBN 9783527328161.

11. H. Langhals, A. J. Esterbauer, A. Walter, E. Riedle and I. Pugliesi, 'Förster resonant energy transfer in orthogonally arranged chromophores', J. Am. Chem. Soc., 2010, 132, 16777-16782. 